\title{
Risk analysis between possible and probable
}

\author{
M. El-Cheikh, A. H. C. Chan \& J. Lamb \\ Department of Civil Engineering, Birmingham University, UK
}

\begin{abstract}
Risk analysis is essential to successful risk management plans and to the best accomplishment of mega construction projects. Probability and possibility theories are the main approaches in analysing risks. However, each theory has its own assumptions, inputs and outputs. This study looks in detail at these two theories and presents an analytical argument to explain the probable concept as a special case of the possible context by considering that a probability percentage is the chance of one possible outcome to occur among several limited possible outputs. Simultaneously, it presents a simple evaluation of Monte Carlo simulation as the main application of the probability theory, and fuzzy sets applications as one of the main applications of possibility theory. In addition, this study also considers whether the risk is probable or possible. Determining the reason behind this uncertainty will definitely help in assigning the proper theory, whether it is probability or possibility, in the decision making stage. Furthermore, and even when a decision is built on the probability theory, the existence of other outcomes should always be considered. This research aims to fill in a few missing gaps in the risk analysis system in order to deliver an improved analysis. The authors argue the high importance of characterizing risks according to their sources so that the expert will be able to classify the risk as either a probable or possible risk in order to be able to choose the theory on which the decision should be based.

Keywords: risk analysis, qualitative risk assessment, quantitative risk analysis, risk management, possibility theory, probability theory, fuzzy set theory, fuzzy logic, construction project.
\end{abstract}

\section{Introduction}

Risk analysis remains one of the biggest concerns for most project managers. This process requires expert knowledge to identify the potential risks and then 
estimate the likelihood and the impact of the severe risks. Risk likelihood and risk impact comprise uncertainty. Analytically, Ang and Tang [9] have been assumed that the total uncertainty is an outcome of randomness and error on prediction. El-Cheikh et al. [3] stated that while previous researches have employed the concept Quantitative Risk Analysis (QRA), they also have demonstrated the application of the probability theory and Monte Carlo simulation or other methods involving fuzzy sets, in order to valorise risks and to introduce a complete risk analysis approach. These studies, however, did not tackle the logic behind the uncertain occurrence of the nominated risks, whether it is the randomness behind this uncertainty or vagueness behind it, consequently there were debates around applying the probability theory, the results from Monte Carlo simulation and applying the possibility theory and fuzzy sets theory.

The organisation of this paper will start with definitions of the probable risks and possible risks, detailed explanations about the probability theory and the possibility theory and the relation between them, presenting the fundamentals behind Monte Carlo simulation and the fuzzy sets theory, to finalise with an appropriate conclusion.

\subsection{Definitions}

Terminologies vary among the different fields, cultures and countries, therefore the author presented in this paper her own definitions of the two main concept about probable and possible risks, taking into consideration that the presented risk analysis method is only related to the civil engineering domain and to the construction projects studies.

\subsubsection{Probable risks definitions}

'Probable Risks' are risks which has neither certain reason behind their occurrence nor certain time or place to occur on; such risks could be associated with any tasks at any time of the project schedule, e.g. protesting against building a prison in Scotland, for several reasons the civilians might protest to stop the engineering work and they might not, and by that the likelihood might be high or small. The impact of such a risk can be high if the protester cut off the way to the site, and it could be very small if they protest in front of the city council against building such a structure in their city. Such types of risks are probable because their impact and likelihood are due to random uncertainty.

\subsubsection{Possible risks definitions}

'Possible Risks' are risks that have a certain reason behind their existence and certain time to occur on, they should be scheduled because they will be allied to a certain activity, e.g. asbestos in the ground soil, the estimator had a geotechnical report about asbestos in the site but the report was not sufficient to demonstrate the size of the asbestos area and depth, therefore the risk of facing asbestos, when the excavation activity is due, has imprecise impact and vague likelihood due to the uncertainty in forecasting which in turn will lead to error in prediction. 


\section{Probability theory}

The last two decades have observed an amazing progress in the use of probability theory as the noticeable successes of this theory were in many applications. Zadeh [13] stated that the successes of this theory are widely recognized, but what is not recognized is a fundamental limitation behind that success. That limitation is the probability theory incapability to function on what Zadeh labeled as perception-based information, for instant, assume that you are looking for a box full of balls, most of these balls are large and only few are small, and this box roughly contains thirty balls, now the question is: What is the probability of pulling out a small ball? The probability theory cannot answer that question simply because there is no mechanism within the theory to present the meaning of perception in a form that provides enough inputs to compute the probability Zadeh confirmed [13]. The author claims that the possibility theory is the one which should be used in this case and the question should be: What is the possibility of pulling out a small ball? She also argues that if there are no limited outputs, certain number of large and small balls in the previous example, the probability theory is inapplicable and it should be replaced by the possibility theory. To understand the probability theory in a better way Vose [12] used the Venn diagrams, which are presented to introduce the basic concepts of probability and possibility theories. Vose [12] explained that:" In a Venn diagram the square border contains all possible events and has an area (probability) equal to unity. The circles present specific events. The area contained by each circle represents the probability that that event could occur". Vose also stated the improbable events are not impossible events. Fig. 1 gives an example of a Venn diagram where two events (A and B) are identified. The events are mutually exclusive meaning that they cannot occur together."

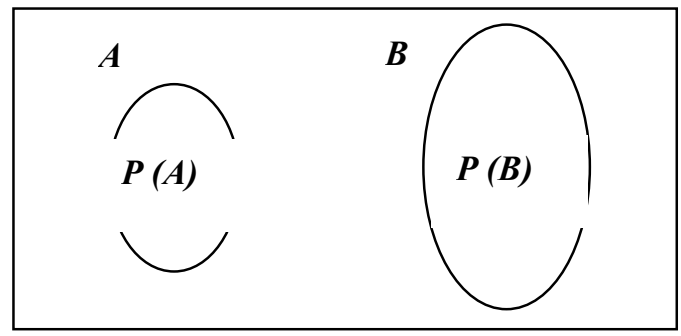

Figure 1: $\quad$ Venn diagram of two events A, B [12].

The author states that if the user is unable to determine the probability of A and $\mathrm{B}$ events as a part of the possible events, she/he will not be able to use the probability theory as she/he could not satisfy its requirements, and that presents another limitation of the probability theory. However, this limitation is not absolute, and in case of the exciting of statistical data, a link between these data and the probability theory could be established to bridge the gap in that theory, 
and that in turn highlighted one of the major reasons behind the significant number of successful applications of the probability theory.

\section{Possibility theory}

Probability and possibility theories are analogous but they are also different, in this paper we will be kooking at the possibilty theory as it is based on the fuzzy sets theory mainly, but as a start a short comparason bettween probability and possibility theories is presented. The main differences between these two theories can be summarized in eqns. (1) and (2) [9]. Eqn. (1) gives that if $p(x)$ is the probability of an $x$ event where $x$ is one possible ouput of the $X$ set of possible outputs then the sum of the probability of all the events is one. e.g. if we toss a coin we will be having two possible outputs: head or tail. The probability of having a head is $50 \%$ and the probability of having a tail is $50 \%$ and the sum of the probability of these two possible outcomes is $100 \%$ or one. While if $\pi(x)$ is the possibility of an event $x$ to occur, then the possibilty of that event alone could be up to $100 \%$ or one. In other words in possibility theory we do consider several possible outcomes on the same time and when a decision will be made, it is built on the highest possibility event. That could be translated by the following example: assume there is a need to establish an access to a new site, so the solution could be either enlarge the existing truck road, establish a new link with the high way, or transform the pathway into an access road or may be some other possible solutions; however, each possible solution has its own possiblity which might be $100 \%$ each or $80 \%$, etc, and this possibility is not affected by any way by the possiblity of the other option, an issue that make the difference in the probable and possible concept more clearer.

$$
\sum_{x \in X} p(x)=1 \text { while } \max _{x \in X} \pi(x)=1
$$

Eqn. (2) implies that the intersection between the possible event $x$ and its complement $\bar{x}$ is not always zero. In eqn. (2) the feature of the fuzzy sets theory started to appear, where there is a shared area between the event $x$ as a set and its complement $\bar{x}$ as a set too as it is shown in fig. 3. This relation will explained in more details in the next paragraph.

$$
\pi(x) \cap \pi(\bar{x}) \neq 0
$$

The relationship between possibility and probability is offered by Zadeh [13] in the "possibility/probability consistency principle What makes it useful is that one often knows a variable's possibility distribution but not its probability distribution: the principle basically says not to assign high probability to a value of $x$ for which there is low possibility. The author claims that such a case happened on rating risks by using the CIRIA method, where there is tendency to 
consider a risk which has low impact and high likelihood equal to a risk which has high impact and low likelihood.

\subsection{Possibility theory based on fuzzy sets}

In this section we will talk about presenting any possible events by using the fuzzy sets theory, but before that a simple definition of the fuzzy concept is presented. Fuzzy logic is unlike the Boolean logic ( 0 or 1 , black or white), it allows multi state partial membership for an element (shades of grey) robust to vague or imprecise, missing data and non-linearity [13]. Presenting a fuzzy element and its membership is delivered by the fuzzy sets theory. Fuzzy sets theory was developed specifically to deal with uncertainties that are not statistical in nature. It allows a computer to model the real world in the same way that human brains do, this theory is different than a traditional set in a way that allows the gradual transition from belonging into not belonging and that is presented by the membership function as it is shown in fig. 2. [9].

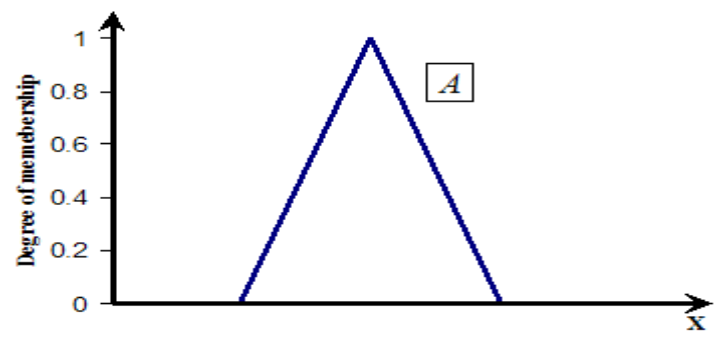

Figure 2: $\quad$ Membership function for fuzzy set A [9].

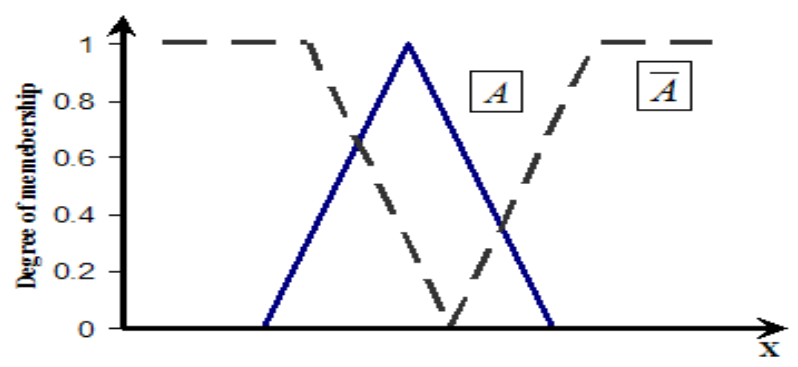

Figure 3: Complement of fuzzy set A [9].

Fig. 2 presents a membership function for triangular fuzzy set $A$, where the horizontal axis could be any parameter, e.g. cost or duration, while the vertical axis presents the degree of that membership, e.g. if the duration of an activity $A$ is a working week approximately, then the values on the horizontal axis will ranged from five to eight days, while the membership values will range from zero to one where the possibility degree of the possible duration cannot be higher then one. 
Fig. 3 presents the relation between a fuzzy set $A$ and its complement $\bar{A}$ and it describes eqn. (2), where the intersection between a possible output and its complement is not necessarily zero, e.g. if activity $A$ was not approximately a week but it was approximately 7 days and by that the values on the horizontal axis will ranged from six to nine days, and finishing the activity in 7 days still possible in the two possible outcomes.

\section{Risk analysis techniques}

Perry and Hayes [8] claimed that in theory, risk analysis techniques have been established for a couple of decades but in practice, the real application of these techniques on construction projects has been restricted, and that is primarily because only multi-disciplinary consultants were able to apply these techniques. From the other hand Flanagan [4] argued that there are no lack on the willingness on using the available risk analysis and management systems but there is a lack of reliable feedback data to provide sufficient inputs into the current risk systems, Flanagan [4] also insisted that these systems are hungry to data and that there is a substantial need to bridge the gap between the shortage of data and the available risk management systems. Another argument is presented bellow to describe three main techniques as they are believed to be the most relevant techniques for better risk analysis and risk management approach.

\subsection{The simple guide CIRIA SP 154}

CIRIA method applies the possibility theory concept; the user needs the CIRIA method to a start a proper risk analysis. Where the user has to establish a list of all the possible risks, then he has to estimate their impact and likelihood to be able later on to rate these risks, and where Risk $=$ Likelihood $*$ Impact in order to be able to focus on major risks. This method is made to help in making proper decisions that could help in minimising risks and dealing with uncertainties [2]. This method also satisfies the Office of Government Commerce (OGC) requirements [7] through presenting a risk register and evaluating the impact and the likelihood of identified risks then developing a strategy to manage their effects. However, this method treats both impact and likelihood on the same weight, and it only presents a qualitative risk analysis guide.

\subsection{Monte Carlo simulation}

Vose [12] defines Monte Carlo simulation as the method, which involves the random sampling of each probability distribution within the module to produce hundreds or even thousand of scenarios (also called iterations or trials). Each probability is sampled in a manner that reproduces the distribution's shape. The distribution of the values calculated for the model outcome therefore reflects the probability of the values that could occur. The principal on whom Monte Carlo simulation is built is the strong law of large numbers that law says that the larger the sample size, the closer their distribution will be to the theoretical distribution. 
In other words, and as Vose [12] explains this law, the greater the number of iterations, the closer the real risk analysis output to the exact distribution of the model's output. Furthermore, the cardinal rule of risk analysis modeling is that: "Every single iteration of a risk analysis model must be a scenario that could physically occur" if the user follows Vose cardinal rule he will have a much better chance in producing a model that is accurate and realistic Vose declared [12]. But the remained question is how to make sure that these iterations, which are usually hundreds, are possible scenarios especially when you are dealing with a project plan that contains hundreds activities. Besides, when the user is choosing a number of iterations, which is usually big, he is assuming that there are certain number of success including in that large number, and so again how many assumptions the user is going to input to Monte Carlo simulation bearing in mind that these assumptions are fundamental to produce accurate and realistic outputs. The author claims that Monte Carlo needs many valid assumptions, and that generates restrictions on the application of Monte Carlo simulation in the construction projects. Furthermore, she argued that for better results from Monte Carlo simulation; the project's plan should be as realistic as possible and that could be achieved either by implementing a sufficient feedback system or by having statistics data, preferably both of them.

\subsection{Fuzzy sets technique}

Applying fuzzy sets theory in risk analysis helps in minimizing the error in the estimations. This concern is believed to be achieved by linking the risk's impact parameter and risk's likelihood parameter.

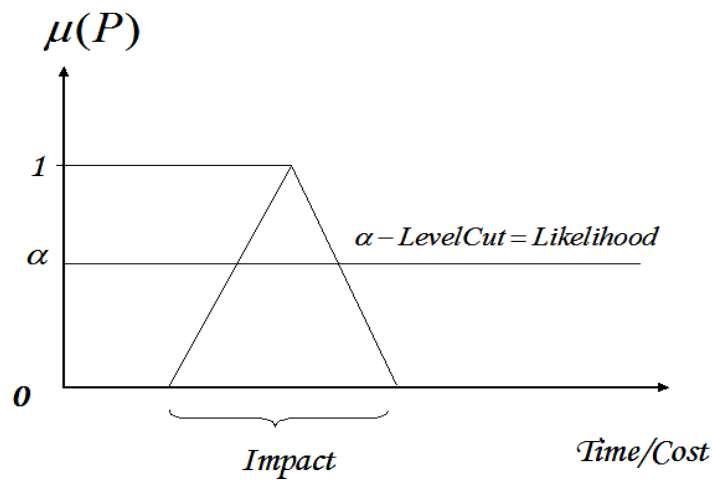

Figure 4: $\quad$ Presenting Risk Impact and Likelihood as a fuzzy set.

The author claimed that treating these two parameters as a fuzzy set will lead to best present to their risk, as it is presented in fig. 4. Where the possible risk is related to a particular activity, then his impact is going to affect either this activity's duration or this activity's cost, and by that impact is the fuzzy parameter on the horizontal axis as it is shown in fig. 4. Since the likelihood of 
that impact is related to the risk's possibility, then this likelihood is presented by the membership degree $\mu(P)$. In other words, since the membership degree presents the degree of believe in that possible element, then that reflects the likelihood of that element. Since the likelihood is the possibility of an element then it will range from zero to one at the maximum and that is presented by the $\alpha$ level cut as it is shown in fig. 4. By that the real impact to the named risk under the estimated possibility is the trapezoidal part of the triangular fuzzy set. In this way of presenting, the fuzzy sets technique helped in rating the risk by presenting its two main components in a logical method. It worth to mention that the centre of that trapezoidal present the most likely duration/cost for activity $A$ under the named risk. Furthermore, applying fuzzy sets theory could help in mastering the project's plan. Mastering the plan is usually achieved by addressing the appropriate links among the project's activities; taking into consideration the uncertainty surrounding the estimation of the tasks durations. The planners try to find the best relations among the tasks in order establish as much realistic plan as possible, but how to do that remains the main problem that faces the planners during scheduling the project.

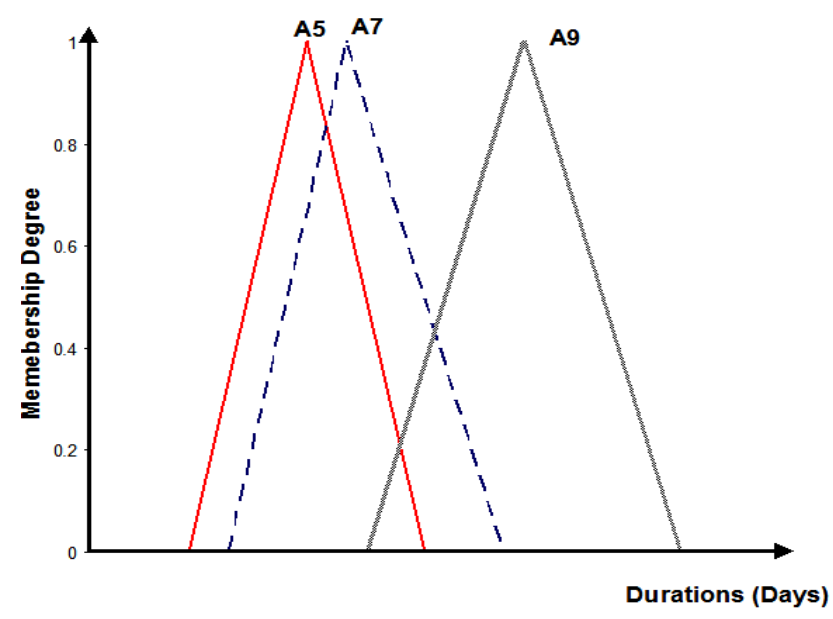

Figure 5: Three activities presented as fuzzy sets.

Through presenting each activity as a fuzzy set the link among the activities will be clearer, and the planner will be able to determine the relationship among these activities. Fig. 5 presented three fuzzy activities A5, A7 and A9, the planner want to know which activity should proceed A5, as we can see in fig. 5 the shared area between A5 and A7 is bigger then the one between A5 and B9 and by that the planner will link A5 with A7 by end-start relation, and A5 with A9 by end-start relation with a certain lag. Additionally, possibility indexes and possibility measures could also be calculated and presented if the planners need 
such a piece of information. However, the application of fuzzy sets theory is explained in more details in $[1,3,6]$, and the reader is referred for these studies for further explanations.

\section{Conclusion}

The successful applications of Monte Carlo simulation should mask the awareness of the probability theory's concept, assumptions and requirements.

In the Civil Engineering domain a never ended debate has been established around the necessity of a risk quantitative analysis method and around adopting Monte Carlo simulation as a main reference. The author argues that the nature behind most of the risks in the construction projects has the possibility nature hence these risks are possible risks not probable, therefore applying the probability theory to quantify these risks is difficult and the alternative could be by applying the possibility theory instead. The possibility theory has huge applications in uncertainty and decision making, and within the absence of statistics data, repetitive conditions and similar circumstances, construction projects lied under the mutually exclusive events, possibility theory based fuzzy sets could present a good alternative to present a better risk analysis in the construction industry in UK.

\section{Acknowledgments}

The authors wish to thank Professor Rogers, the head of Infrastructure Engineering and Management Research Center at the University of Birmingham, for the financial assistance and the anonymous referees for their careful reading of the manuscript and their fruitful comments and suggestions, and Dr. Nicholas Gorst for his work on the research. Special mention also to Mr. Mike Wilkes and Mr. Alex Buchan at INTERSERVE Project.

\section{References}

[1] Ayyub, Bilal M. and Haldar, Achintya. Project Scheduling Using Fuzzy Set Concept. Construction Engineering \& Management, 110 (2), pp.189204, 1984.

[2] The CIRIA Organisation, CIRIA SP154. A Simple Guide to Controlling Risk. London. www.ciria.org/acatalog/sp154.pdf

[3] El-Cheikh M, Lamb J, Gorst N. Risk Quantitative Analysis Using Fuzzy Sets Theory. Proc. of the Int. Conf. of Computational Methods in Sciences and Engineering, eds T. Simos \& G. Maroulis, VSP: Leiden, pp. 159-162, 2004.

[4] Flanagan, R., The Hong Kong Polytechnic University Web Site, Managing Risk for Uncertain Future- A project Management perspective. http://bsun04.bre.polyu.edu.hk/rccree/events/pm_symposium/RogerFlana gan.pdf 
[5] Lam, K.C., So, A.T.P., Hu, Tiesong, Ng, Thomas, Yuen, R. K. K., Lo, S. M., Cheung, S. O., and Yang, Hongwei . An Integration of the Fuzzy Reasoning Technique and the Fuzzy Optimization Method in Construction Project Management Decision-Making. Construction Management \& Economics, 19 (1), pp.63-76, 2001.

[6] Lorterapong, P. and Moselhi, O. (1996). Project Network Analysis Using Fuzzy Sets Theory. Construction Engineering and Management, 122 (4), pp. 308-318, 1996.

[7] Office of Government Commerce, Risk and Value Management, Crown: London, 2003.

[8] Perry, J. G. and Hayes, R. W., Risk and its Management in Construction Projects. Proc. of the institution of Civil Engineers. Engineering Management Group, Thomas Telford: London, pp. 499-521, 1985.

[9] Ross, Timothy J. and Lucero, Jonathan L. (eds). Fuzzy Logic and Probability applications, Bridging the Gap, ASA- SIAM Series on Statistics and Applied Probability: Philadelphia, Alexandria, Virginia. SIAM \& ASA, 2002.

[10] TAH, J.H.M and CARR, V., A proposal for construction project risk assessment Using Fuzzy Logic. Construction Management \& Economics, 18(4), pp. 491-500, 2000.

[11] Dimitrov, V. and Korotkich, V., (eds). Fuzzy Logic, a Framework for the New Millennium, Studies in Fuzziness and Soft Computing. New York, 2002.

[12] Vose, D., Risk Analysis A Quantitative Guide, John Wiley \& Sons Ltd: England, 2000

[13] Zadeh, A. Lotfi, Fuzzy Sets. In Klir, G. and Yuan, B. (eds). Fuzzy Sets, Fuzzy Logic, and Fuzzy System, World Scientific Publishing Co Pte Ltd: Singapore, NJ and London, 1996. 